Moss, G. \& Barnea, Z. (1976). J. Appl. Cryst. 9, 510-511. National Bureau of Standards (1964). Monograph 25, Section 3, p. 21.

National Bureau of Standards (1976). Monograph 25, Section 13, p. 35.

Prager, P. R. (1971). PhD thesis, Univ. of Melbourne, Australia.

REID, J. S. (1983). Acta Cryst. A39, 1-13.

Stevenson, A. W., Gao, D., Pain, G. N. \& Wieluński, L. S. (1991). Acta Cryst. A47, 128-133.

Stevenson, A. W. \& Pain, G. N. (1990a). J. Appl. Phys. 68, 569-573.

Acta Cryst. (1993). A49, 183-190
Stevenson, A. W. \& Pain, G. N. (1990b). Aust. J. Phys. 43, 793-799.

Stevenson, A. W., Wilkins, S. W., Kwietniak, M. S. \& Pain, G. N. (1989). J. Appl. Phys. 66, 4198-4200.

TANNER, B. K. (1989). J. Electrochem. Soc. 136, 3438-3443.

WII KINS, S. W. (1978a). Proc. R. Soc. London Ser. A, 364, 569-589.

WILKINS, S. W. (1978b). Acta Cryst. A34, 343-344.

Wooster, W. A. \& Macdonald, G. L. (1948). Acta Cryst. 1, $49-54$.

ZACHARIASEN, W. H. (1945). Theory of X-ray Diffraction in Crystals. New York: Wiley.

\title{
On Predicting Scan Profiles: the Nature of the 'Aberration Function'
}

\author{
By Riccardo Destro \\ Dipartimento di Chimica Fisica ed Elettrochimica, Università di Milano, Via Golgi 19, Milano 20133, Italy \\ ANi Richard E. Marsh
}

The Beckman Institute, ${ }^{*}$ California Institute of Technology, Pasadena, California 91125, USA

(Received 11 February 1992; accepted 15 July 1992)

\begin{abstract}
In an earlier treatment [Destro \& Marsh (1987). Acta Cryst. A43, 711-718], an attempt was made to predict the shapes of high-angle $\theta-2 \theta$ scan profiles by convoluting a low-angle profile with the presumedly known spectral distribution function for the incident (crystal-monochromatized Mo $K \alpha$ ) radiation but it was found necessary to introduce a third component, an 'aberration function', that varied with the Bragg angle $\theta$. It is shown here that the primary purpose of the aberration function is to correct for defects in the spectral-distribution function. In particular, the effective intensity ratio between the $K \alpha_{2}$ and $K \alpha_{1}$ spectral lines can apparently deviate greatly (by more than $10 \%$ ) from the theoretical value of 0.499 , depending upon the alignment of the monochromator crystal, and an appreciable amount of white radiation may also be present. By a suitable modification of the spectral-distribution function, high-angle scan profiles can be predicted from accurate measurements of a low-angle profile; as a result, scan-truncation losses can, for the most part, be removed. However, modeling the spectral distribution function appropriate to a particular experiment remains a difficult empirical procedure.
\end{abstract}

\section{Introduction}

The problem of scan truncation in single-crystal diffractometry is well known: at even moderately high

* Contribution No. 8573.

0108-7673/93/010183-08\$06.00
Bragg angles, the angular range in a typical $\theta-2 \theta$ scan is usually not large enough, owing to interference with neighboring reflections, to encompass the entire scan profile. Consequently, the tails beyond the scan range are missing from the recorded intensity and the measured backgrounds, being recorded within these tails, are artificially large; if these backgrounds are subtracted from the total scan intensities in the usual way, the net intensities will again be systematically underestimated. It appears that, taken together, these two effects can lead to recorded intensities that are too low, perhaps by $15 \%$ at $2 \theta=40^{\circ}$ (Destro \& Marsh, 1987; hereinafter, DM).

In an attempt to arrive at a simple procedure for estimating the amount of this 'truncation loss' in a particular experiment, DM considered the possibility that the entire scan profile at any Bragg angle might be predicted from a single profile measured at a very low angle (where the entire profile can be captured). In this development, DM first presumed that the intensity profile at any angle is a combination (a convolution) of two components: (1) a known $\theta$ dependent component, representing the emission spectrum of the incident radiation; and (2) an unknown $\theta$-independent component, reflecting other characteristics of the incident beam and the size and mosaicity of the diffracting crystal. In principle, this second, $\theta$-independent, component (which DM called the 'basic profile') could be derived by deconvoluting, from a measured low-angle profile, the emission spectrum appropriate to that angle; indeed, at a low enough angle $\left(2 \theta<10^{\circ}\right.$ or so $)$, the emission spectrum would be close enough to a delta function

(C) 1993 International Union of Crystallography 
that an experimental profile measured at such an angle could be taken directly as the basic profile. Profiles at higher angles could then be predicted by convoluting the basic profile with the known spectral distribution.

Unfortunately, when DM tested this procedure by comparing the predicted high-angle profiles with experimental profiles covering expanded scan ranges, they found a lack of satisfactory agreement, particularly in the important regions within the tails; to reproduce the experimental profiles it was necessary to introduce a third, $\theta$-dependent, component which DM called an 'aberration function'. This made the procedure for predicting profiles and truncation errors more complicated: it was apparently necessary to record with great care not only a low-angle reflection (to obtain the basic profile) but also a relatively large number of higher-angle reflections at various Bragg angles and covering as wide a scan range as possible to obtain a reliable mapping of the aberration function. Recently, we have made further studies in attempts to understand the source of the aberration function, with the ultimate hope of circumventing it. This paper is the result of those attempts.

\section{The nature of the aberration function}

Following Alexander \& Smith (1962), Ladell \& Spielberg (1966), Mathieson (1982) and others, we represent the intensity profile in single-crystal diffractometry as the convolution of two types of functions, the first, $I_{b}$ (the 'basic profile'), including all nondispersive terms (independent of Bragg angle) such as the source and receiving aperture sizes and the crystal mosaicity, and the second, $I_{\lambda}$, including all dispersive terms, the most important (and, presumably, only) one being the spectral distribution characteristic of the incident radiation. We then write

$$
I(\beta)=I_{b}(\beta) * I_{\lambda}(\beta, \theta),
$$

where $I$ is the profile intensity as a function of $\beta$, the deviation from the Bragg angle $\theta$. When intensities are measured by $\theta-2 \theta$ scans, the intensity profile at any Bragg angle $\theta$ will contain an additional term, $T(\beta)$, representing its truncation due to the finite scan range, so we write

$$
I^{\prime}(\beta)=T(\beta)\left[I_{b}(\beta) * I_{\lambda}(\beta, \theta)\right],
$$

where $T(\beta)$ is a truncation (or window) function equal to unity within the scan range and equal to zero elsewhere. However, to match the experimental profiles at all Bragg angles, DM found it necessary to introduce the 'aberration function' $a(\beta, \theta)$, writing

$$
I_{\exp }=a *\left[T\left(I_{b} * I_{\lambda}\right)\right] .
$$

The aberration function appropriate for any angle $\theta$ was derived by deconvoluting $I^{\prime}$ from $I_{\text {exp }}$ following Stokes's (1948) procedure, which makes use of the
Fourier transforms of the various terms; details of this procedure, including some caveats, are given in DM.

Typical aberration functions, obtained by applying this procedure to a large number of profiles measured for a crystal of syn-1,6:8,13-biscarbonyl[14]annulene* (hereinafter BCA; see Destro, 1988) and averaging within $10^{\circ}$ ranges, are represented in Fig. 1. The first 27 terms of the Fourier transforms of the aberration functions are plotted; the real and imaginary terms are shown separately. Departures of the real terms from 1.0, and of the imaginary terms from 0.0 , represent the modifications to the theoretical profile $I^{\prime}$ needed to match the experimental profile $I_{\text {exp }}$.

The aberration function can account for the failure either of $I_{b}$ to represent the nondispersive component adequately or of $I_{\lambda}$ to represent the dispersive component adequately or of both. During exchanges relating to reflection shapes, Mathieson $(1989 a, b)$ expressed interest in attempting to identify the physical origin of the aberration functions. To assist in this attempt, copies of the Fourier-transform components of these functions for BCA, extending out

\footnotetext{
* IUPAC name: tricyclo[4.8.1.1 $\left.{ }^{8,13}\right]$ hexadeca-1,3,5,7,9,11,13heptaene-15,16-dione.
}
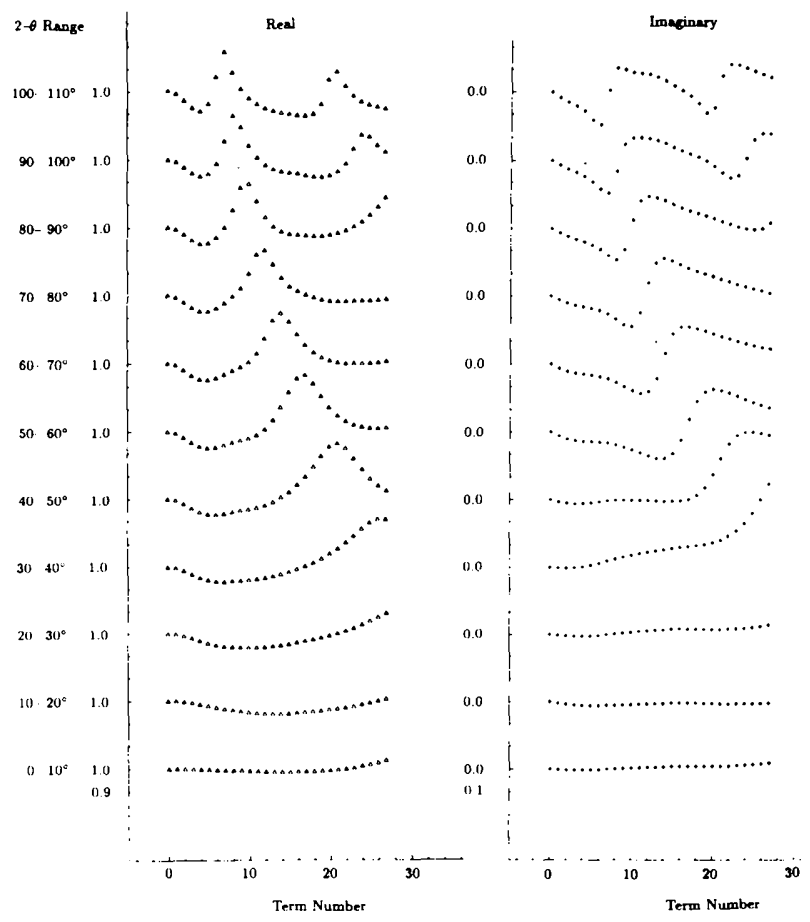

Fig. 1. Fourier-transform terms of the aberration functions obtained at different $2 \theta$ angles for crystals of BCA, obtained by deconvoluting experimental profiles at various $2 \theta$ values with the idealized profile $I^{\prime}(\beta)$. Scale markings along the ordinates are at 0.1 intervals. 
to 50 points, were sent to him in April 1989. Later in that year, Mathieson reported that he obtained a straight line when he plotted the reciprocal wavelength of the wave-like features of the Fourier transforms (see Fig. 1) $v s \tan \theta$, implying that the $\theta$ dependence of the aberration functions was the same as that of the wavelength dispersion. Accordingly, it seemed probable that the most important features of the aberration function served to correct for errors in the assumed spectral-distribution function $I_{\lambda}$. In the following section, we describe our efforts to understand what these errors might be.

\section{The true shape of $\boldsymbol{I}_{\boldsymbol{\lambda}}$}

In principle, $I_{\lambda}$ might be obtained directly by deconvoluting an experimental high-angle profile with a low-angle one (representing the basic profile). However, the nature of the functions makes this impossible, since the two intensity functions being deconvoluted have similar and rather broad shapes from which one cannot obtain with any reliability the high-frequency terms necessary to represent correctly the sharp spectral distribution. We note that the same difficulty arises, in principle, when we obtain an aberration function by deconvoluting an experimental profile with the 'idealized' profile $I^{\prime}(\beta)$, since both profiles have similar shapes. The difference is that, in analyzing the aberration functions, we can concentrate on the low-order Fourier terms of the transforms, since these terms provide the important general features of the inadequacies in the spectral distribution function; the higher-order Fourier terms (which cannot be accurately obtained by the deconvolution process) represent high-frequency ripples that, we believe, have little significance. On the other hand, to reproduce the entire spectral-distribution function faithfully by deconvoluting a high-angle profile with the basic profile, these high-order terms are essential. The 30 or so low-angle Fourier terms necessary to understand the aberration functions can be determined with confidence by the deconvolution process; the several hundred terms necessary to generate a true spectral distribution curve cannot.

Accordingly, we have investigated $I_{\lambda}$ in a more indirect way, as follows: (1) obtain a basic profile $I_{b}$ from a low-angle reflection; (2) convolute it with the assumed spectral-distribution function $I_{\lambda}$ appropriate to a particular Bragg angle; (3) compare the result with the experimental profile $I_{\exp }$ measured at that angle; (4) on the basis of the comparison, attempt to make reasonable modifications to $I_{\lambda}$. We have carried out this procedure on two different crystals; since the results differed somewhat, we report them separately.

The first crystal, A, was of BCA (Destro, 1988), ground to an approximate sphere of diameter $0.35 \mathrm{~mm}$; it was cooled to $16 \mathrm{~K}$ (to avoid thermal diffuse scattering). The second crystal, $B$, was of glycine and was also approximately spherical (of diameter $0.5 \mathrm{~mm}$ ); it was cooled to $23 \mathrm{~K}$. Profiles were measured on two different modified Syntex diffractometers, those for BCA at Milano and those for glycine at Pasadena. Features common to the two diffractometers are the low-temperature Samson cryostat (Samson, Goldish \& Dick, 1980), Mo radiation and the perpendicular configuration of the monochromator crystal $\left(\varphi_{i}=270^{\circ}\right.$ for BCA and $90^{\circ}$ for glycine, in the notation of Mathieson, 1968, Fig. 2); differences included the type of X-ray tube (Philips for BCA, Seifert for glycine, both normal focus), the operating voltage and current $(50 \mathrm{kV}$ and $30 \mathrm{~mA}$ for BCA, $45 \mathrm{kV}$ and $20 \mathrm{~mA}$ for glycine) and the (fixed) diameter of the circular receiving aperture $(2.5 \mathrm{~mm}$ for BCA, $2.0 \mathrm{~mm}$ for glycine). Profiles were collected with a $2 \theta$ scan range of $\left(3.4+S_{\alpha_{1}, \alpha_{2}}\right)^{\circ}$ for BCA and of $\left(4.0+S_{\alpha_{1}, \alpha_{2}}\right)^{\circ}$ for glycine, where $S_{\alpha_{1}, \alpha_{2}}$ is the $K \alpha_{1}$, $K \alpha_{2}$ separation. The basic profile $I_{b}$ for BCA was from the $\overline{1} 01$ reflection at $2 \theta=5.94^{\circ}$; for glycine, 020 at $6.91^{\circ}$. The basic profile for the glycine crystal was appreciably broader than for BCA (full width at half-maximum, FWHM, 0.41 vs $0.30^{\circ}$ ), presumably because of the larger crystal size. Other details of the experimental arrangements, including a description of the method of normalizing the profiles and removing the intrinsic background, are given by DM and by Destro (1988). There resulted several hundred background-free normalized profiles, from which we selected those with $2 \theta \geq 90^{\circ}: 100$ for BCA and 61 for glycine; in both cases, approximately one-half of the profiles were in the range $90 \leq 2 \theta \leq 100^{\circ}$ and the other half in the range $100 \leq 2 \theta \leq 110^{\circ}$. The spectral line shape $I_{\lambda}$ was taken as the standard double-Lorentzian function described by DM [equations (4) and (5)], with the assumptions that $\lambda_{\alpha_{1}}=0.70930, \lambda_{\alpha_{2}}=$ $0.71359 \AA$ (Bearden, 1967), $\Delta \lambda_{1}=0.29, \Delta \lambda_{2}=$ $0.32 \mathrm{xu}(\mathrm{Mo}) \quad\left[1 \mathrm{xu}(\mathrm{Mo})=1.0021 \times 10^{-13} \mathrm{~m}\right]$ and a ratio $I_{\alpha_{2}} / I_{\alpha_{1}}$ of 0.499 (Compton \& Allison, 1935).

In the comparison of the calculated high-angle profiles $I_{b} * I_{\lambda}$ with the measured profiles, we found it convenient to define several quantitative features: (1) $R_{1}$, the ratio between the $K \alpha_{2}$ and $K \alpha_{1}$ peak intensities; (2) the separation between these two peaks, which we describe by $\lambda_{K \alpha_{2}}$, the apparent wavelength of $K \alpha_{2}$ relative to an assumed value of $0.70930 \AA$ for $K \alpha_{1}$; (3) $W_{1}$ and $W_{2}$, the FWHM of each of these peaks; (4) $I_{1}$ and $I_{96}$, the intensities at the two scan extremes, i.e. at the first and last of the 96 profile steps; (5) $R_{2}$, the ratio of these two intensities $\left(I_{1} / I_{96}\right)$. The matching of these features of $I_{b} * I_{\lambda}$ with those of $I_{\exp }$ is surely a necessary condition but, perhaps, not a sufficient condition for the removal of the aberration function. Since all these quantities, particularly (2), (4) and (5), are sensitive to statistical fluctuations in the individual profile measurements, our results are averaged over the many profiles we recorded for both crystals. 
Table 1. Averaged values of some relevant features of high-angle experimental profiles $I_{\text {exp }}$ compared with those of the corresponding idealized profiles $I_{b} * I_{\lambda}$ (see text)

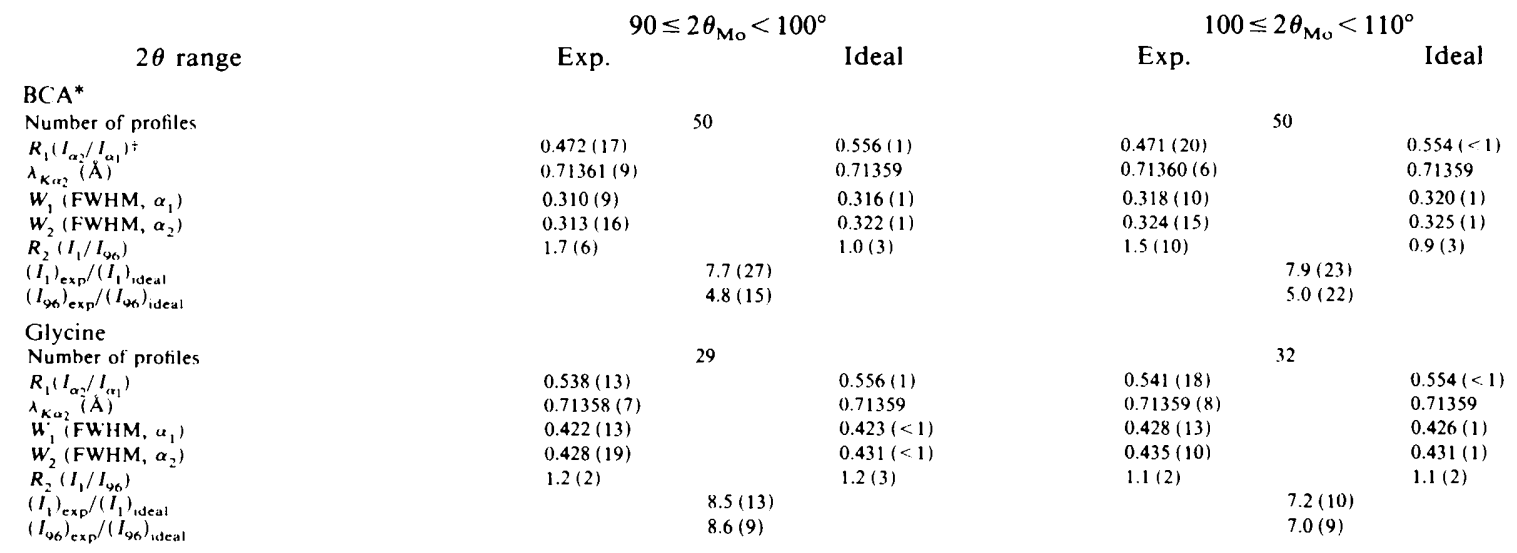

* syn-1,6:8,13-Biscarbonyl[14]annulene (Destro, 1988).

+ This ratio is for the full profile at the indicated $2 \theta$ range; it is not to be confused with the spectral-distribution ratio, whose theoretical value is 0.499 .

Results are given in Table 1, where we compare these five features as calculated from $I_{b} * I_{\lambda}$ with the averaged experimental profiles $I_{\text {exp }}$ in the two different scan regions and for the two different crystals. A number of observations can immediately be made.

(1) The peak-height ratio $R_{1}$ for the calculated profiles, about 0.555 , is appreciably larger than the theoretical spectral-distribution ratio of 0.499 ; this is due to the convolution with the relatively broad basic profile. However, the same ratio of 0.555 is obtained for both crystals, even though the basic profile for glycine is appreciably broader than for BCA (see above).

(2) $R_{1}$, the intensity ratio of $K \alpha_{2}$ to $K \alpha_{1}$, is much lower than predicted for the observed profiles of BCA, but only slightly lower than predicted for the glycine profiles.

(3) The experimental values of the wavelength separation, expressed as $\lambda_{K \alpha_{2}}$, agree well with the theoretical values.

(4) The experimental peak widths $W$ also agree, within their scatter e.s.d.'s, with the theoretical values.

(5) The experimental intensities at the scan extrema, $I_{1}$ and $I_{96}$, are notably higher than predicted, in all cases.

(6) The ratios $R_{2}$ between the intensities at the two extrema are larger than predicted for BCA, but not for glycine. This is probably a consequence of the second effect: that, in the case of BCA, the apparent intensity of $K \alpha_{2}$ relative to $K \alpha_{1}\left(R_{1}\right)$ was considerably smaller than the theoretical value of 0.555 .

These observations suggest two distinct effects: the effective spectral distribution showed an intensity ratio $K \alpha_{2}$ to $K \alpha_{1}$ different from the theoretical value of 0.499 , by different amounts for the two crystals; and the effective spectral distribution has broader tails than represented by the double-Lorentzian func- tion $I_{\lambda}$, almost certainly due to a small but significant amount of white radiation and to the well known deviations between any type of Lorentzian model and the experimental shape of an X-ray line (see, for example, Hoyt, 1932). It appeared, then, that a major role of the aberration function is to correct for these two defects in the theoretical spectral-distribution function. In attempts to understand these defects more fully, we have investigated the situation in a somewhat more direct manner, using deconvolution processes.

As noted earlier, the true spectral distribution cannot be obtained by direct deconvolution of an experimental profile by a basic one because of Fourier limitations. However, by considering only the lowerorder Fourier terms and thus introducing severe ripples into the resulting functions, we can obtain pseudo spectral-distribution curves that can be compared quantitatively. We found that, when taking the Fourier transforms of the basic and the experimental profiles, only the first 40 or 50 terms (real and imaginary) were significant, i.e. able to pass the acceptance criteria based on estimated variances and empirical cut-off limits (see DM). Accordingly, we performed the deconvolution of $I_{\exp }$ with $I_{b}$ using only these 40 or 50 terms and then compared the resulting spectral-distribution function with a function obtained by first transforming the theoretical function $I_{\lambda}$ into its Fourier components and then reconstituting it from the same number of terms as was used in the deconvolution of the experimental profiles. We thus obtained two pseudo spectral-distribution functions, theoretical and experimental, both highly distorted because of the limited number of Fourier components but which can be compared with each other in quantitative detail.

The modified spectral-distribution curves calculated from these truncated Fourier series differ from 
the real distributions in two important ways (in addition to the ripples caused by the truncation): the peak intensity ratio $K \alpha_{2}$ to $K \alpha_{1}$ is altered and the peak widths are greatly expanded. For example, when we calculate the pseudo spectrum at a $2 \theta$ value of $106.2^{\circ}$, corresponding to the $6,18, \overline{16}$ reflection from $\mathrm{BCA}$, we find that the peak intensity ratio is altered from the value 0.499 for a standard theoretical distribution spectrum to 0.562 for the modified pseudo spectrum and the peak widths are expanded from 0.062 to $0.189^{\circ}$ for $K \alpha_{1}$ and from 0.070 to $0.182^{\circ}$ for $K \alpha_{2}$. When we then derive the experimental pseudo spectral curve for $6,18, \overline{16}$, we find similar peak widths, perhaps slightly narrower for $K \alpha_{1}$ and slightly broader for $K \alpha_{2}$, but a very different ratio of peak heights, 0.50 rather than 0.562 . This is the same trend as indicated in Table 1 and strongly suggests that the effective intensity ratio $K \alpha_{2}$ to $K \alpha_{1}$ in the spectral-distribution function $I_{\lambda}$ was about $11 \%$ smaller than the nominal value of 0.499 in the case of BCA and about $3 \%$ smaller in the case of glycine.

A comparison of the modified spectral-distribution curve obtained from the $6,18, \overline{16}$ reflection of BCA
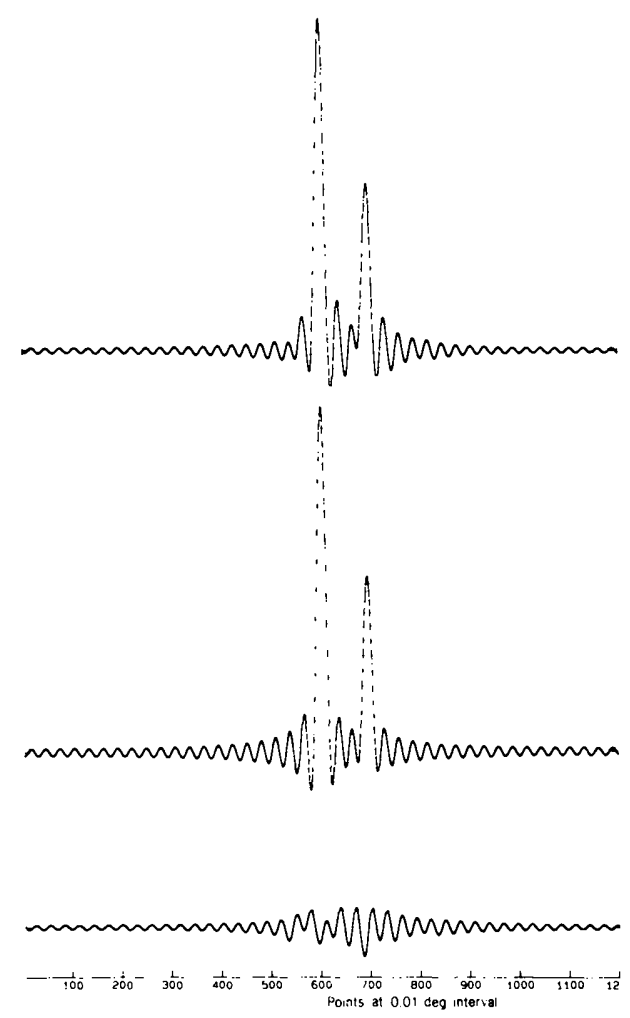

Fig. 2. Modified spectral-distribution functions at $2 \theta=106.2^{\circ}$. (Top) Experimental, obtained from the first 41 Fourier terms resulting from the deconvolution of the measured profile of the $6,18, \overline{16}$ reflection from BCA with the 'basic profile'. (Center) Theoretical, obtained by the reconstruction, again from the first 41 Fourier terms, of a model spectral distribution in which the $K \alpha_{1}$ to $K \alpha_{2}$ intensity ratio was taken to be 0.44 . (Bottom) Their difference. with the pseudo spectral distribution calculated for the same $2 \theta, 106.2^{\circ}$, and with a value of 0.44 for the $K \alpha_{2}$ to $K \alpha_{1}$ intensity ratio is shown in Fig. 2. The reduction of the $K \alpha_{2}$ to $K \alpha_{1}$ intensity ratio seems to have been a major step in the right direction; however, the difference curve at the bottom of Fig. 2 shows important features. A careful comparison of the curves suggests that many of these features are related to the symmetry of the $K \alpha_{1}$ and $K \alpha_{2}$ peaks; that is, the peaks of the experimental pseudo spectral function (Fig. 2, top), while of the same height and width as those of the theoretical double-Lorentzian function (Fig. 2, center), are asymmetric with respect to them. It seems likely that this asymmetry effect may arise from the same source that leads to the large change (in the case of BCA) in the apparent $K \alpha_{2}$ to $K \alpha_{1}$ intensity ratio: alignment of the monochromator crystal. If this very delicate alignment is such as to discriminate in favor of the $K \alpha_{1}$ line (that is, if the acceptance function of the monochromator is not centered midway between the two peaks), asymmetry will be introduced into the peak shapes. The amount of asymmetry will depend upon the shape of the acceptance function. Less can be said about the nature of the broader tails of the experimental profiles (Table 1) or as to how to model the white radiation that is implied.

\section{The role of the basic profile}

The question remains as to whether the aberration function could be due, in part, to deficiencies in the basic profile. Throughout our investigations we have noted that different low-angle reflections, from the same crystal and with approximately the same Bragg angle, showed small but significant differences in their profiles, due, perhaps, to anisotropic mosaicity effects. In addition, these basic profiles were collected at Bragg angles that, while small, were not identically zero; thus, some small dispersion effects might be present. We have investigated the possible importance of these two effects by examining Fourier transforms of the aberration functions obtained by deconvoluting high-angle reflections with various theoretical profiles $I_{b} * I_{\lambda}$ formed from different basic profiles $I_{b}$.

For this purpose, 50 high-angle profiles $(100 \leq$ $2 \theta_{\mathrm{Mo}} \leq 110^{\circ}$ ) of BCA were used as experimental models. For each of them, four ideal profiles $I_{b} * I_{\lambda}$ were calculated from four different basic profiles $I_{b}$ : (1) $b_{1}$, from the $\overline{1} 01$ reflection of BCA, as described earlier; (2) $b_{1 d}$, obtained by deconvoluting from $b_{1}$ the standard double-Lorentzian description of the theoretical spectral distribution; (3) $b_{2}$, from the 101 reflection of $\mathrm{BCA}$, at $2 \theta=6.41^{\circ}$; this reflection showed a FWHM of $0.29^{\circ}$, slightly narrower than that for $\overline{1} 01$, $0.30^{\circ}$, and it also had a noticeably different shape in the tail regions; (4) $b_{2 d}$, again deconvoluted as in $b_{1 d}$. We note that, at $2 \theta=6^{\circ}$, the $K \alpha$ peaks are very sharp 
(FWHM $=0.0024^{\circ}$ for $\alpha_{1}$ and $0.0027^{\circ}$ for $\alpha_{2}$ ) and the separation of the $\alpha_{1}-\alpha_{2}$ doublet for Mo radiation is about $0.036^{\circ}$, almost exactly the size of a single step in our 96 -step procedure for measuring profiles $\left(3.44^{\circ}\right.$ total scan range for both $b_{1}$ and $b_{2}$ ). Thus, the deconvolution could be performed by a simple algorithm of the type

$$
P^{\prime}(n)=P(n)-\frac{1}{2} P^{\prime}(n-1),
$$

where $n$ is the step number and $P(n)$ the background-

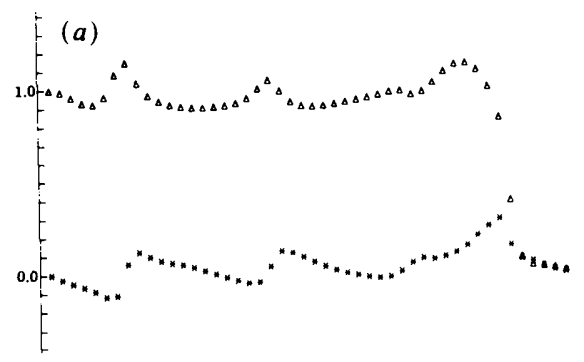

(b)

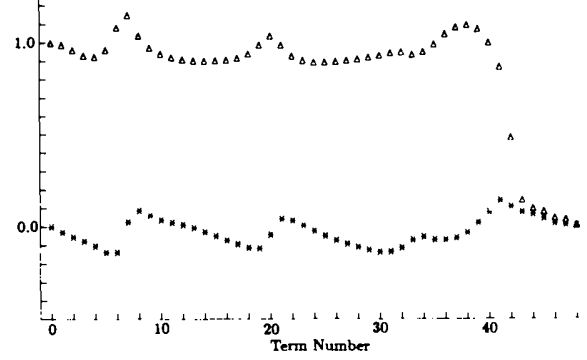

(c)

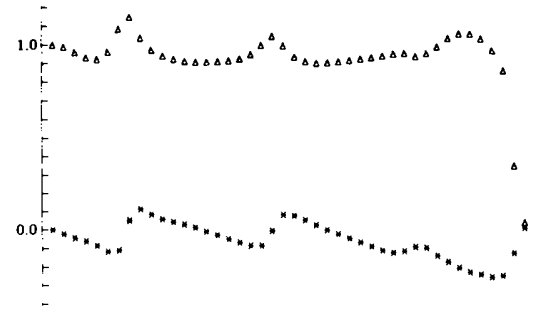

(d)

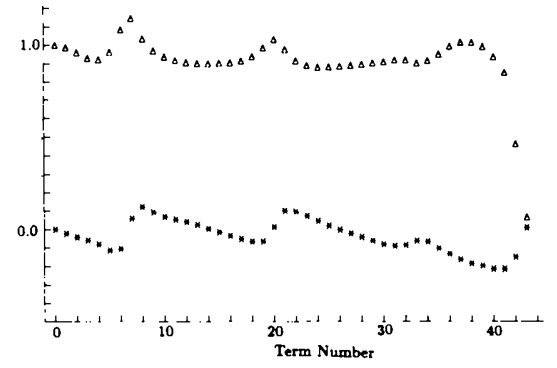

Fig. 3. Fourier transforms of aberration functions for BCA at $100 \leqslant 2 \theta_{\mathrm{Mo}_{\mathrm{o}}} \leq 110^{\circ}$, calculated from four different basic profiles: $(a)$ the 101 reflection, unmodified; $(b) \overline{101}$ with the standard (double-Lorentzian) spectral-distribution function deconvoluted; $(c)$ the 101 reflection; $(d)$ 101, modified as in $(b)$. free observed profile $b_{1}$ or $b_{2}$; in other words, we assume two delta functions, having the ratio $2: 1$ and separated by one step. This procedure avoids the undesirable oscillations that would be created by the usual Fourier methods. In both cases the deconvolution reduced the widths of the basic profile by 0.001 $0.002^{\circ}$.

Convolution of each of these four basic profiles with the standard double-Lorentzian function $\left(I_{\lambda}\right)$, followed by the truncation $T(\beta)$, generated four sets of 50 idealized profiles; subsequent deconvolution from the corresponding experimental profiles yielded Fourier transforms of the aberration function, which were then averaged for the 50 reflections. The results are shown in Fig. 3.

As we had anticipated, a change of the basic profile did not have a great effect on the aberration-function transforms. Replacement of the basic profile of $\overline{1} 01$ with that of 101 leads to minimal changes in the real Fourier terms but to noticeable changes in the imaginary terms [compare Fig. 3(a) with 3(c) or Fig. $3(b)$ with $3(d)]$. Similarly, compressing the basic profiles by removing the spectral term $I_{\lambda}$ [curves $(b)$ and $(d)$ in Fig. 3] had an appreciable effect only on the imaginary terms. None of the changes had a significant effect on the most prominent wave-like features of the aberration function, the features we attribute to defects in the spectral-distribution function $I_{\lambda}$.

\section{The white-radiation effect}

The comparison between experimental and idealized profiles, as summarized in Table 1, clearly indicated the presence of white radiation in the spectral distribution. We could not arrive at a direct method for estimating the amount (and shape) of this white radiation, so resorted to a trial-and-error technique. We performed a series of tests, first on a limited number of data and then on our entire set of 649 measured profiles for BCA crystals, each test consisting of (1) the inclusion of a (different) amount of white radiation (in addition to a modification of the $K \alpha_{2} / K \alpha_{1}$ ratio) in the representation of $I_{\lambda},(2)$ the calculation of an idealized profile $I_{b} * I_{\lambda}$, taking 101 as the basic profile $I_{b}$ and (3) the comparison of the result with an experimental profile at the same value of $2 \theta$, with particular emphasis placed on the first and last profile steps. We represented the white radiation at first as a constant contribution to the spectral intensity but found improvement by adding a slope term that increases the contribution at the low-angle portion of the spectral range relative to the high-angle portion. However, this latter process had the side effect of altering the $K \alpha_{2}$ to $K \alpha_{1}$ intensity ratio in the synthesized profiles. Thus, at this point, the procedure became an empirical trial-and-error one. 


\section{Concluding remarks}

The results of our efforts here are perhaps best shown in Fig. 4, a before-and-after treatment of a typical profile. At the top left of Fig. 4 is shown the experimental scan profile of a moderate-angle reflection from BCA, $11,0,11$ at $2 \theta=69.45^{\circ}$, superposed with a synthetic profile obtained by a convolution of the basic profile $\left(b_{2 d}\right.$, above) with the theoretical spectral distribution $I_{\lambda}$, unmodified in any way; to its right is the Fourier transform of the aberration function obtained by deconvoluting these two profiles. The curves at the bottom of Fig. 4 are similar, except that the spectral distribution has been modified in two ways: (1) the intensity ratio of $K \alpha_{2}$ to $K \alpha_{1}$ has been changed from the theoretical value of 0.499 to 0.42 ; (2) a white-radiation component has been added, totalling about $0.1 \%$ of the total $K \alpha_{1}$ intensity and about three times as large at the low-angle edge as at the high-angle edge. The improvement from top to bottom is marked. Moreover, this improvement is due only to the modifications to the spectral distribution curve: the change in the $K \alpha_{2}$ to $K \alpha_{1}$ intensity ratio and the addition of the white-radiation background. The major and rather sharp features of the original aberration functions (Fig. 4, top; see also Fig. 1) are due, almost totally, to the effective $K \alpha_{2}$ to $K \alpha_{1}$ intensity ratio being other than the theoretical value of 0.499 ; these features nearly disappeared when the intensity ratio was modified to 0.44 . On the other hand, the white-radiation background is evidenced, in the aberration functions, primarily in the lowfrequency terms: the rather shallow dip in the 'real' curve (Fig. 4, top right) presumably measures the total amount of white radiation, and the difference between the low-angle and the high-angle background must be included in the 'imaginary' terms.

Thus, the concept of a 'basic profile', obtained from a low-angle reflection, seems entirely valid; from this basic profile and from a detailed analysis of accurately measured high-angle profiles, modifications to $I_{\lambda}$ can be made, complete profiles can be derived for any angle $\theta$ and truncation errors can be calculated with confidence. However, the modification process of this spectral-distribution function $I_{\lambda}$ is delicate, the different adjustments interacting with one another in many ways. Moreover, the appropriate adjustments apparently change from one experiment to another, as demonstrated by the quite different values we obtained for the apparent $K \alpha_{2}$ to $K \alpha_{1}$ intensity ratio for glycine and for BCA. It seems quite certain that monochromator alignment is a key factor. In this regard, physical reality might be better served if a 'monochromator function' could be generated as a

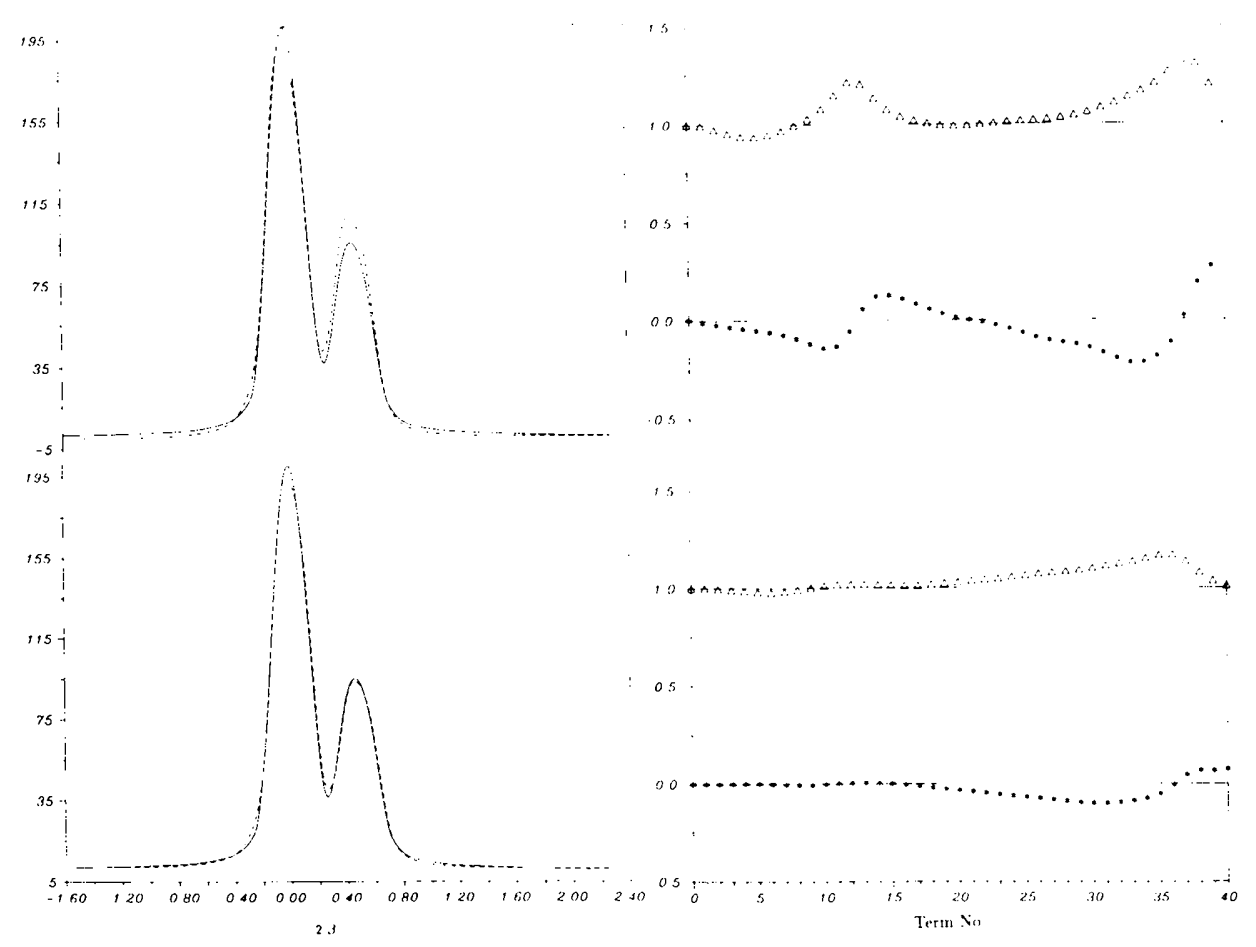

Fig. 4. Left: Observed (solid line) and synthetic (dashed) scan profiles of the $\overline{11}, 0,11$ reflection from BCA. The synthetic profiles were calculated from the basic profile, $\overline{1} 01$, convoluted (top) with the unmodified spectral-distribution function $I_{\lambda}$; (bottom) with a modified spectral distribution having the intensity ratio of $K \alpha_{2}$ to $K \alpha_{1}$ equal to 0.42 and with a background term added. The horizontal coordinate $2 \beta$ is the deviation from the reference angle $2 \theta$, taken as the center of $K \alpha_{1}$. Right: the corresponding aberration functions (Fourier components), derived by deconvoluting the experimental curves with the synthetic ones. 
substitute for our 'aberration function'; this monochromator function would describe the intensity passed by the monochromator as a function of wavelength, including 'band-pass' limits at the two ends and could thus have the effect both of altering the effective $K \alpha_{2}$ to $K \alpha_{1}$ intensity ratio and of adding a white-radiation component that is so clearly needed.

We emphasize that all our experiments have been made at low temperature $(20 \mathrm{~K}$ or less) and we have assumed thermal diffuse scattering to be negligible. At higher temperatures, TDS becomes appreciable, perhaps being as large as, and in the opposite sense from, truncation losses. Conceivably, then, one could end up worse off by correcting room-temperature data for truncation while ignoring TDS. But our work on truncation is based on the fact that, for careful work in such areas as electron-distribution studies and highly precise bond-length determinations, lowtemperature data are of utmost importance (particularly for molecular crystals), not only to avoid TDS but also to permit the accurate determination of the displacement parameters $U_{i j}$ that is afforded by high-angle intensity data. For such experiments, truncation losses are extremely important: an intensity loss of $15 \%$ at $2 \theta=40^{\circ}$ for Mo radiation corresponds to an apparent increase in the isotropic component of the atomic displacement coefficient $U$ of over $0.004 \AA^{2}$. Further efforts to arrive at convenient and reliable methods of correcting for such losses are clearly warranted.

\section{Afterword}

At the suggestions of thoughtful referees, we add a few comments.

(1) We emphasize that the 'basic profile' depends on the characteristics (size, mosaic character, etc.) of the particular crystal being studied; it would be dangerous and unjustified to use the basic profile for one crystal in analyzing the high-angle profiles of another.
(2) Our recommendations for low-temperature measurements apply to molecular crystals. For many inorganic systems with high Debye temperatures, measurements at or near room temperature might well be adequate.

(3) Our experimental data were, as we have noted, obtained with the monochromator in the perpendicular orientation. For instruments with a parallel configuration, expressions for the spectral resolution would need to be modified.

(4) An interesting experiment, which we have not carried out, would be to measure the band-pass characteristics of the monochromator, perhaps (as suggested by Mathieson, $1989 a$ ) by scanning the profile using a very narrow slit.

(5) We regard this work as developmental; while $\mathrm{RD}$ has devised many computer programs to carry out the various calculations, these programs are being continuously revised and have not been thoroughly debugged. Thus, they are not available for distribution.

We are indeed indebted to Professor A. McL. Mathieson for many helpful and insightful comments.

\section{References}

Alexander, K. E. \& Smith, G. S. (1962). Acta Cryst. 15, 983-1004.

Bearden, J. A. (1967). Rev. Mod. Phys. 39, 78-124.

COMPTON, A. H. \& Allison, K. S. (1935). X-rays in Theory and Experiment, pp. 640, 745. New York: Van Nostrand.

Destro, R. (1988). Aust. J. Phys. 41, 503-510.

Destro, R. \& MARSH, R. E. (1987). Acta Cryst. A43, 711-718.

Hoyt, A. (1932). Phys. Rev. 40, 477-483.

Ladell, J. \& Spifl.berG, N. (1966). Acta Cryst. 21, 561-567.

Mathieson, A. MCL. (1968). Rev. Sci. Instrum. 39, 1834-1837.

Mathieson, A. MCL. (1982). Acta Cryst. A38, 378-387.

Mathieson, A. MCL. (1989a). Acta Cryst. A45, 613620.

MATHil:SON, A. MCL. (1989b). Private communication.

Samson, S., Goldish, E. \& Dick, C. J. (1980). J. Appl. Cryst. $13,425-432$.

Stokes, A. R. (1948). Proc. Phys. Soc. London Sect. A, 61, 382-391.

Acta Cryst. (1993). A49, 190-198

\title{
Energy-Dispersive Diffuse X-ray Scattering Technique. I. Principles
}

\author{
BY JOHN S. REID \\ Department of Engineering, Fraser Noble Building, The University, Aberdeen AB9 $2 U E$, Scotland
}

(Received 23 May 1992; accepted 7 August 1992)

\begin{abstract}
Energy-dispersive diffuse $\mathrm{X}$-ray scattering is a particularly appropriate technique for use with high-
\end{abstract}

0108-7673/93/010190-09\$06.00 energy synchrotron-radiation sources. Its multiplex and geometric advantages are outlined and the comparatively simple principles behind the technique are detailed. The energy-dispersive scan spreads scatter-

(C) 1993 International Union of Crystallography 